\title{
ALICE ITS: the Run 1 to Run 2 transition and recent operational experience
}

\section{Domenico Colella*}

European Organization for Nuclear Research (CERN)

Institute of Experimental Physics, Slovak Academy of Sciences (IEP SAS)

E-mail: domenico.colella@cern.ch

for the ALICE Collaboration

\begin{abstract}
The characterisation of the Quark-Gluon Plasma produced in ultra-relativistic heavy-ion collisions is the main goal of the ALICE experiment at the CERN LHC. The ALICE Inner Tracking System (ITS) plays a key role in the study of short-living hadrons through the primary and secondary vertex reconstruction. The system is composed of two innermost layers of pixel detectors, two middle layers of drift detectors and two outermost layers of strip detectors. The ITS covers the central pseudo-rapidity range of $|\eta|<0.9$ and the distance from the beam line ranges from $3.9 \mathrm{~cm}$ for the innermost layer to $43 \mathrm{~cm}$ for the outermost layer. During the Run 1 data taking period, the ITS contributed with tracking and charged particle identification capabilities, as well as a contribution to the definition of the Level 0 trigger signal. In particular at low $p_{\mathrm{T}}$ the event reconstruction relies on the ITS performance as tracks do not reach the outer tracking detectors. In this contribution, after a brief description of the design of the three sub-detector systems and the respective performance during Run1, the consolidation activities carried out during the LHC long shutdown period (LS1) and the re-commissioning to prepare the detector for the data taking during Run2 will be presented.
\end{abstract}

24th International Workshop on Vertex Detectors

1-5 June 2015

Santa Fe, New Mexico, USA

${ }^{*}$ Speaker. 


\section{Introduction}

ALICE (A Large Ion Collider Experiment) [1] is the LHC experiment optimized for the study of the strong interacting matter created in heavy-ion collisions. The fundamental theory of strong interaction, Quantum Chromo-Dynamics (QCD), predicts that a phase transition to a plasma of deconfined quarks and gluons, the Quark-Gluon Plasma (QGP), occurs at energy densities above $1 \mathrm{GeV} / \mathrm{fm}^{3}$ that can be achieved in nucleus-nucleus collisions at the LHC energy [2].

The ALICE apparatus has a high detector granularity, a low transverse momentum cut off of $p_{\mathrm{T}} \approx 0.1 \mathrm{GeV} / c$, and good particle identification (PID) capabilities up to $20 \mathrm{GeV} / c$. The detector subsystems can be classified in three groups: a central barrel, covering the full azimuth in the pseudo-rapidity region $|\eta| \leq 0.9$, the muon spectrometer placed in the forward pseudo-rapidity region $(-4.0<\eta<-2.5)$ and the forward detectors positioned in the high pseudo-rapidity region (small angles with respect to the beam line). The central-barrel detectors are embedded in the L3 solenoid magnet, which provides a field up to $0.5 \mathrm{~T}$ along the beam direction, and aim to investigate the particle production at mid-rapidity. The forward detectors address e.g. the measurement of photons and charged particles in the forward region, deliver the time and the longitudinal position of the interaction and are used for triggering and for the determination of the centrality in $\mathrm{Pb}-\mathrm{Pb}$ collisions. The muon spectrometer is used to measure heavy-flavour, quarkonium and light vector meson production in the forward region.

The experiment continuously recorded data during the first physics campaign of the machine from fall 2009 until early 2013 and is now involved in the LHC Run2 campaign. Besides running with $\mathrm{Pb}$ ions, for the assessment of the properties of the created aforementioned matter, the physics programme includes also the study of $\mathrm{pp}$ and $\mathrm{p}-\mathrm{Pb}$ collisions. These control measurements, besides being interesting in themselves, are fundamental references for the comprehension of the underlying collision dynamics allowing to separate the genuine QCD-matter signals from the cold-matter initial- and final-state effects.

\section{ALICE Inner Tracking System}

The Inner Tracking System (ITS) [3] and the Time Projection Chamber (TPC) are the main tracking detectors in the central barrel. The ITS is a cylindrically-shaped silicon tracker that surrounds the interaction region and is composed of six tracking layers of silicon detectors, namely two layers each of Silicon Pixel Detectors (SPD), Silicon Drift Detectors (SDD) and double-sided Silicon Strip Detectors (SSD) (Fig. 1).

The ITS was designed to improve the position, angle, and momentum resolution for tracks reconstructed in the TPC, whose inner radius is $80 \mathrm{~cm}$, to identify the secondary vertices from the decay of hyperons and heavy-flavoured hadrons, to reconstruct the interaction vertex with a resolution better than $100 \mu \mathrm{m}$, and to recover particles that are missed by the TPC due to acceptance limitations. The number, position and segmentation of the layers and the chosen technology were optimized for efficient track finding and high impact-parameter resolution in the severe highmultiplicity environment expected for central $\mathrm{Pb}-\mathrm{Pb}$ collisions at LHC energy. In particular, the outer radius was driven by the necessity to match tracks reconstructed using only the ITS information with those reconstructed in the large volume TPC. The inner radius is the minimum allowed 


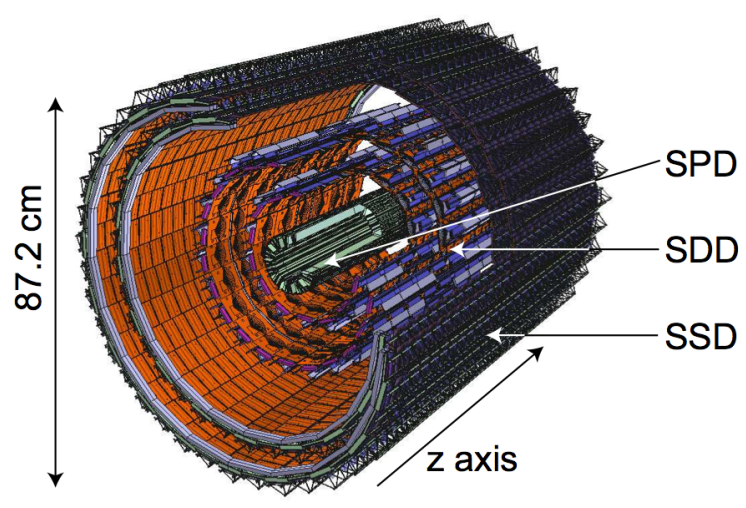

Figure 1: Layout of the ITS.

by the radius of the beam vacuum tube. In addition to tracking, the SDD and the SSD provide charged-particle identification via the measurement of the specific ionization energy loss $\mathrm{d} E / \mathrm{d} x$; this feature gives the ITS standalone capability as a low $p_{\mathrm{T}}$ particle spectrometer. Moreover, the SPD contributes to the definition of the Level 0 trigger of the experiment.

The SPD modules consist of a two-dimensional sensor matrix with 40960 cells of reversebiased silicon detector diodes, with $200 \mu \mathrm{m}$ thickness. Two modules are mounted together along the $\mathrm{z}$ direction to form a half-stave, and two mirrored half-staves are attached, head-to-head along the $\mathrm{z}$ direction, to a carbon-fiber-composite support sector. Each sector hosts six staves: two on the inner layer and four on the outer layer. In total, the SPD includes 60 staves, consisting of 240 modules with 1200 read-out chips. The read-out is based on a binary concept, where the full matrix is shifted out on a 32 bit bus. In addition to the individual pixel hit recording, each pixel chip generates a pulse whenever at least one pixel cell detects a particle signal above the threshold. This pulse is used to implement a prompt trigger which contributes to the Level 0 trigger.

The SDD modules are mounted on linear structures called ladders, for a total of 260 modules, assembled in such a way to ensure full angular coverage. One SDD module consists of a drift detector and its front-end electronics. The sensitive area of a detector is split into two drift regions, where electrons move in opposite directions, by a central cathode kept to a nominal voltage of $-1800 \mathrm{~V}$.

The SSD sensor is a double-sided silicon strip detector mounted on carbon-fiber support structures, with a strip pitch of $95 \mu \mathrm{m}$, a stereo angle of $35 \mathrm{mrad}$ and having $768 \mathrm{P}$ - and $768 \mathrm{~N}$-side strips. The detector modules, each consisting of one sensor connected to two hybrids for the signal read-out, are assembled in 72 ladders, each one having 22 to 25 modules on layer 5 and 34 to 38 modules on layer 6 , resulting into 1698 modules. The detector is optimized for low mass, with material budget $\mathrm{X} / \mathrm{X}_{0}$ less than $1 \%$, in order to minimise multiple scattering.

A detailed description of the ITS as well as its commissioning with cosmic rays and LHC beams can be found in [4].

\section{ITS performance during Run1}

The whole ITS has been operational during RUN 1 of LHC; a summary of the active time of 


\section{Running Time (hours)}

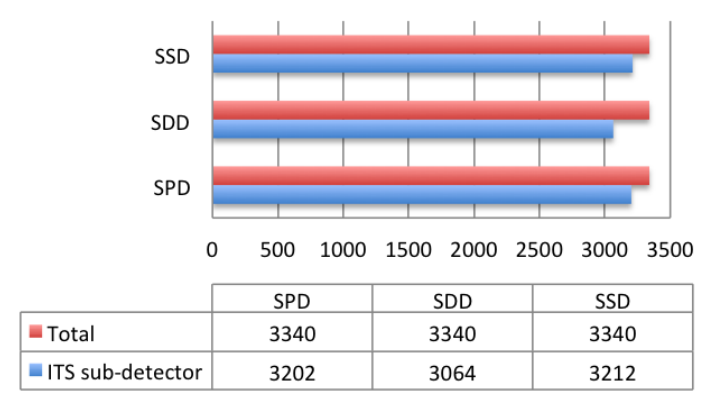

EOR reasons

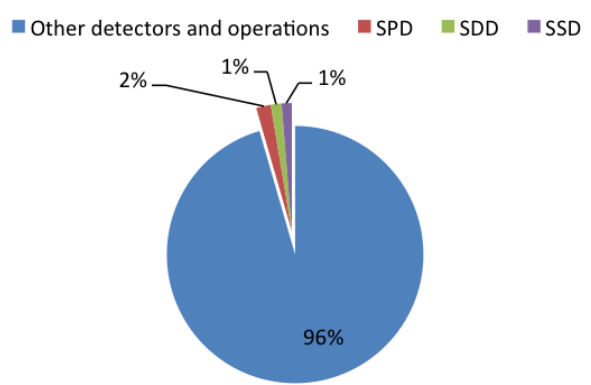

Figure 2: Running statistics for the three ITS sub-detectors during the LHC Run1 period.

the three sub-detectors is shown in Fig. 2.

All the three detectors took data for more than $90 \%$ of the total ALICE data taking time, having been in less than $4 \%$ of the cases the reason for the stopping of a run. For the SPD and the SDD the most probable cause of stopping the run was a communication loss between the frontend and the read-out electronics with a consequent assertion of a busy status. Data format errors occurred in very few occasions for the SDD and the SSD, for the latter interpreted in some cases as a consequence of single event upsets $\left(\mathrm{SEU}^{1}\right)$.

The coverage of the geometrical acceptance has been quite stable for the SDD and SSD for all Run1, with $87 \%$ and $91 \%$ of the modules active respectively. In the case of the SPD, due to the problems with the cooling system, the percentage of active modules initially decreased to a minimum of $63 \%$, then started to increase, thanks to the corrective action described in Section 4, reaching 93\% of active modules in the $\mathrm{p}-\mathrm{Pb}$ period at the beginning of 2013.

A comprehensive and detailed description of the performance of the ALICE experiment during Run1 is reported in [5]. In the following the attention is focused on the ITS performance.

The reconstruction of the interaction vertex position is one of the key tasks that the ITS has to accomplish. Three different algorithms were developed to perform this task and two of them rely on the usage of local SPD reconstruction by coupling pairs of points in the first two layers of the detector (tracklet). The third method is based on an iterative procedure that allows to obtain a precise measurement of the primary vertex, using tracks reconstructed in the whole ALICE central barrel with the ITS and the TPC. The reconstruction of the primary vertex by the SPD is the first step in the full track reconstruction, that is performed via a Kalman filter algorithm. The procedure requires, after the reconstruction of the tracks in the TPC, the prolongation to the ITS, starting from the matching with the outermost SSD layers and continuing through the SDD, down to the SPD. To extend the $p_{\mathrm{T}}$ acceptance down to $\approx 0.1 \mathrm{GeV} / c$ and to recover tracks not reconstructed in the TPC, an ITS-standalone tracking algorithm was developed. The efficiency of the prolongation of the ITS standalone tracks to the TPC, if tracks with at least two points in the ITS are considered, is above $95 \%$ and is almost constant for all momenta.

The resolution of the track impact parameter $\left(\mathrm{d}_{0}\right)$, computed as the distance of closest approach of a given track to the vertex, is a benchmark to define the tracking precision of the system.

\footnotetext{
${ }^{1} \mathrm{SEU}$ - Single Event Upset, the change of the state of memory cells or transistors caused by ionizing radiation.
} 

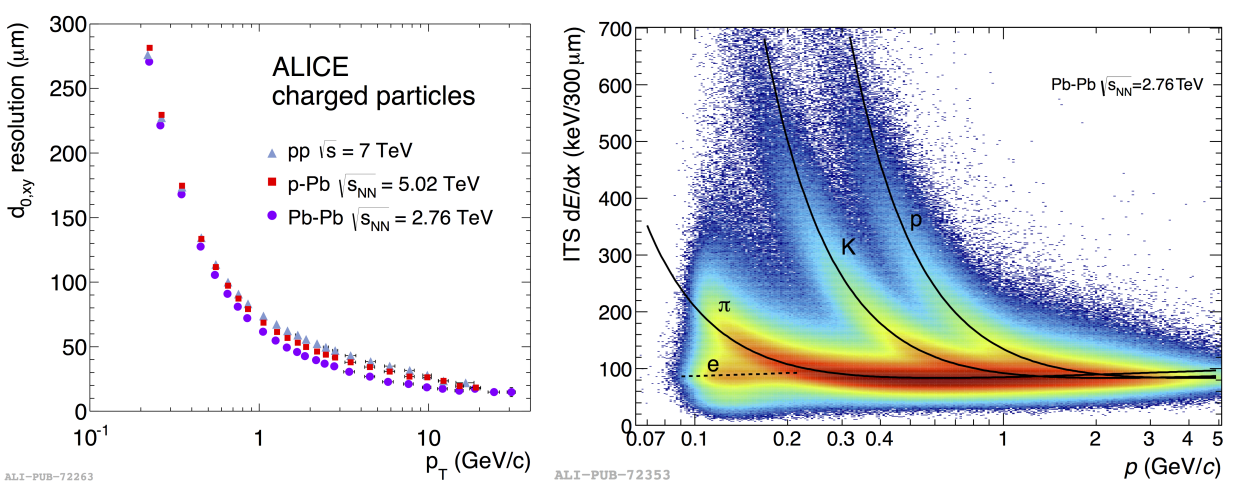

Figure 3: Left: Transverse impact parameter resolution for charged particles as a function of $p_{\mathrm{T}}$ for different colliding systems. Right: $\mathrm{d} E / \mathrm{d} x$ for charged particles as a function of their momentum measured by the ITS in standalone mode in $\mathrm{Pb}-\mathrm{Pb}$ collisions at $2.76 \mathrm{TeV}$.

The left panel in Fig. 3 shows the resolution of the transverse distance to the primary vertex for identified ITS-TPC tracks for three colliding systems; the contribution from the vertex resolution is not subtracted. One can notice an improvement of the resolution in heavier systems as a result of the higher particle multiplicities that allow a more accurate determination of the interaction vertex. The precise measurement of the track impact parameter and the capability to separate the secondary vertices originating from heavy-flavour decays are fundamental requirements for the reconstruction of heavy-flavour particle decays.

The ITS contributes also to the improvement of the transverse momentum resolution for tracks reconstructed in the TPC; the inverse- $p_{\mathrm{T}}$ has value less than $0.0015(\mathrm{GeV} / c)^{-1}$ for $1 / p_{\mathrm{T}}$ less than $0.1(\mathrm{GeV} / \mathrm{c})^{-1}$.

As anticipated the last four layers of the ITS provide particle identification in the $1 / \beta^{2}$ region based on the specific energy deposit per unit length by a particle, which depends for a given momentum only on its charge and rest mass. The simultaneous measurement of track momentum and signal amplitude in a sensitive detector volume allows one to identify particles. The four SDD and SSD layers provide analogue read-out signals proportional to the charge deposited in each detector. The two signals are combined to obtain a single $\mathrm{d} E / \mathrm{d} x$ value and a truncated mean method is applied to account for the long tails of the Landau distribution. An example of the mean energy deposition as a function of the momentum measured in a $\mathrm{Pb}-\mathrm{Pb}$ collision is shown in the right panel of Figure 3. The particle identification in the ITS combined with standalone tracking allows to identify pions down to a minimum momentum of $p_{\mathrm{T}} \sim 0.1 \mathrm{GeV} / c$ reducing the systematic uncertainty on the yield and the mean transverse momentum measurements due to the extrapolation to $p_{\mathrm{T}} \approx 0 \mathrm{GeV} / c[6]$.

\section{Main interventions during LS1 and readiness for Run2}

In April 2013, after the end of the period dedicated to $\mathrm{p}-\mathrm{Pb}$ collisions, the Run 1 ended and the LS1 period started. This long period, dedicated to the machine and detectors improvements, ended in December 2014. All the three sub-systems of the ITS took advantage of this period to consolidate the systems and fix the few issues that occurred during Run1. 


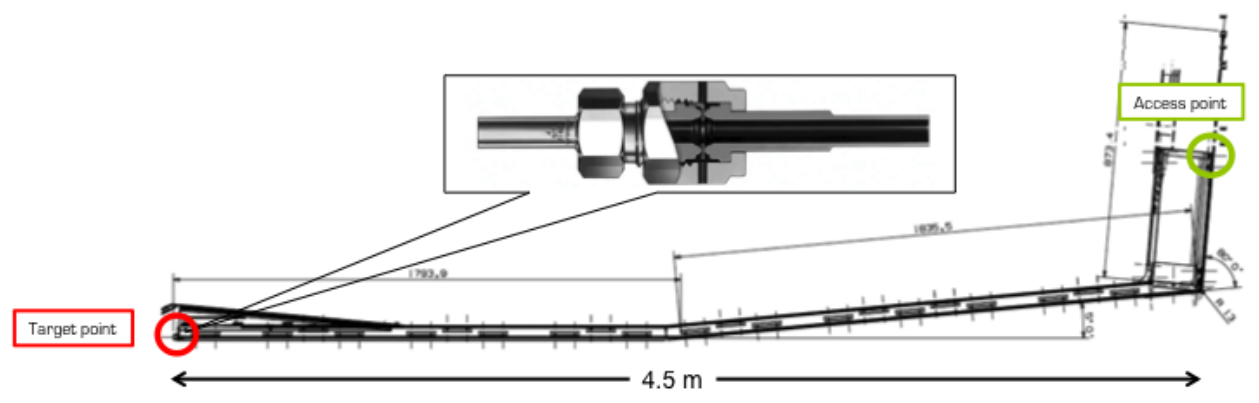

Figure 4: Path of the cooling pipe between the clogged filters (bottom left) and the last accessible point (top right).

Since the installation, the SPD was steadily operated, although a sizeable fraction of modules had been progressively turned off due to a lack of cooling flow underneath. In 2011 the number of modules that could be operated was $63 \%$. The cooling performance was studied in detail; the cause of the local lower cooling efficiency, which prevented the full operation of several modules, was found to be due to insufficient flow of the liquid freon due to partial clogging of the filters installed in the feeding lines very close to the detector, which can only be accessed by dismounting and removing all central barrel detectors. The corrective action to overcome this problem without affecting the other detectors was to drill the filters, in order to re-establish the correct flow of cooling. The drilling procedure was particularly challenging, because the last accessible point before the clogged filters is located at a distance of $4.5 \mathrm{~m}$ from it, as shown in Fig. 4 and the inner diameter of the cooling pipe is only $4 \mathrm{~mm}$. A dedicated tool, constituted by a tungsten carbide tip welded on a stainless steel wire $5 \mathrm{~m}$ long and $2.5 \mathrm{~mm}$ thick, was assembled to perform the intervention. A long cleaning procedure was also carried out, to remove all the fragments generated by the drilling. After this operation the nominal flow of $2.1 \mathrm{~g} / \mathrm{s}$ was established on all 10 cooling lines, well above the value of $1.8 \mathrm{~g} / \mathrm{s}$ that is the minimum value required for a total drain of the heat in case all the half-staves of a sector are powered. During 2012 and the $\mathrm{p}-\mathrm{Pb}$ run in 2013, only 5\% of the detector, i.e. 6 half-staves, was off due to high temperature, including 2 half-staves with a bad thermal contact with the cooling pipe present since the installation of the SPD in ALICE.

The read-out and data transmission system of the whole SDD is mounted close to the sensors and is based on three ASICs: the PASCAL chip that provides both the preamplification and the digitization of the analog signal from the sensor, the AMBRA chip that is a multi-event buffer to reduce the needed bandwidth for the data transmission without increasing the average detector dead time, and the CARLOS chip that performs the zero-suppression and the compression of the data before their transmission via $100 \mathrm{~m}$ long single mode optical fibers by means of the Gigabit optical link to CARLOSrx data processing board. The CARLOSrx is a 10-layers 9U VME printed circuit board, placed in the counting room, with the main task of controlling and receiving data from twelve CARLOS chips, then packing, buffering and after sending them towards the data acquisition system (DAQ). The evidence shows that most of the EOR reason caused by the SDD comes from errors in the CDH. Almost 60\% of the resources usage on the three Xilinx Virtex II-Pro FPGAs was engaged and no space for further improvements in the firmware was available. On top of this, the installed FPGA wasn't anymore supported by ISE design suite, after the release of the software 
version 10, then the decision to build a new board, called SuperCARLOSrx, was taken and the boards were produced in 2011. The SuperCARLOSrx implements a more powerful hardware with five Xilinx Virtex 5 FPGAs and the new feature to work as a common mode filter. All the 24 boards were replaced in April 2013 and tested with the detector during the LS1. The performance of the new board allows the implementation of a new firmware able to handle a high level common mode functionality and a better data compression, reducing the occurrence of $\mathrm{CDH}$ errors.

The 768 strips of one side of a SSD sensor are connected to a hybrid circuit carrying six daisychained HAL25 read-out chips, which amplify and shape the signal. The hybrids are connected to the EndCap boards, which use two ASICSs to decouple the analog and control signals from the sensor bias voltage and buffer all signals to and from the front-end chips and generate the control signals for read-out of the analog buffers using two ASICs. The signals from the 1698 sensors are digitized in the Front End Read-Out Modules (FEROM), which also performs the zerosuppression and offset correction, the event-data de-randomization using Multiple Event Buffers (MEB) and the event data transfer to the DAQ. Differently with respect to the SPD and SDD, the read-out electronics of the SSD is installed in the cavern, just outside the magnet, housed in eight $6 \mathrm{U}$ VME crates, and consequently are inaccessible during beam-time. Because of this, much emphasis was put on high reliability design techniques. Nevertheless during Run1, eight SEUs were identified: four during the $\mathrm{pp}$ collisions and four during the $\mathrm{p}-\mathrm{Pb}$ data taking period. A prediction for the presence of SEUs during Run2, based on an extrapolation of the integrated luminosity, prospects the possibility to have one SEU per day during the pp and 0.04 SEU per day during the $\mathrm{Pb}-\mathrm{Pb}$ period. To reduce the chance of occurrence, the PROMs were upgraded to a radiation tolerant model and a new procedure to reload the FPGA firmware was implemented to speed up the recovery. Other solutions were explored, such as to shield the crates with iron, but discarded because too much expensive or not resolutive.

\section{Conclusions and Outlook}

During the first three year of data taking, all three sub-systems of the ITS were operated succesfully and performed according to expectations in $\mathrm{pp}, \mathrm{p}-\mathrm{Pb}$ and $\mathrm{Pb}-\mathrm{Pb}$ collisions. The ITS played a crucial role in the ALICE data analysis, contributing to the reconstruction of the primary interaction vertex, improving the momentum and angular resolution of tracks reconstructed in the TPC. Operating in standalone mode the ITS extended tracking and PID capabilities of the experiment, allowing the measurement of the transverse momentum spectra for the light-flavour particles (pions, kaons and protons) down to $p_{\mathrm{T}} \sim 0.1 \mathrm{GeV} / c$ [6]. The few hardware problem observed during the Run1 operations have been properly addressed during the LHC LS1. Development of the Detector Control System software and the firmware of the read-out electronics has been carried out to be compliant with the recent improvements of the central systems.

Since the beginning of the LHC Run2 period in January 2015, all three sub-systems of the ITS have been involved in the data-taking campaign with cosmic rays needed for alignment studies. The configuration and the performance of the ITS have been tested during the machine exercises with transfer line test, as well as quiet and non-quiet proton beams at $\sqrt{s}=0.9 \mathrm{TeV}$, on April $5^{\text {th }}$, and at $\sqrt{s}=13 \mathrm{TeV}$, on May $21^{s t}$. The official pp collision period started on June $3^{\text {rd }}$, beginning with $50 \mathrm{~ns}$ beam bunch spacing then moving in August to $25 \mathrm{~ns}$ beam bunch spacing. During 
these first three months, all three detectors took data continuously: the SPD cooling plant is now stable resulting in 111 (out of 120) half-staves active with only one half-stave out because of high temperature, no CDH errors have been observed for the SDD and only one suspected SEU has been seen for the SSD.

In order to improve the ITS physics performance and in particular to extend heavy-quark studies to the decays of charmed baryons and beauty hadrons, an upgrade of the system intended for Run3 is under development [7][8].

\section{References}

[1] ALICE Collaboration, "The ALICE experiment at the CERN LHC", JINST 3, S08002 (2008)

[2] F. Karsch, "Lattice QCD at high temperature and density", Lect. Notes Phys., 583 (2002)

[3] ALICE Collaboration, "Technical Design Report of the Inner Tracking System (ITS)", CERN-LHCC-99-012 (1999)

[4] V. Manzari, "The ALICE Inner Tracking System: commissioning and running experience", PoS (Vertex 2009) 005 (2009)

[5] ALICE Collaboration, "Performance of the ALICE experiment at the CERN LHC", Int. J. Mod. Phys. A 29 (2014)

[6] ALICE Collaboration, "Production of pions, kaons and protons in pp collisions at $\sqrt{s}=900 \mathrm{GeV}$ with ALICE at the LHC", Eur. Phys. J. C 71, 1655 (2011)

ALICE Collaboration, "Pion, Kaon, and Proton Production in Central $\mathrm{Pb}-\mathrm{Pb}$ Collisions at $\sqrt{s_{\mathrm{NN}}}=2.76 \mathrm{TeV} "$, PRL 109, 252301 (2012)

[7] ALICE Collaboration, "Letter of Intent for the Upgrade of the ALICE Experiment", CERN-LHCC-2012-012, LHCC-I-022 (2012)

[8] M. Kofarago, "Upgrade of the Inner Tracking System of ALICE", in proceedings of this conference. 